\title{
Journal of Natural \& Ayurvedic Medicine
}

\section{Standardization of Herbal Drugs: A Critique}

\author{
*Nanjan MJ \\ University of Madras, India
}

*Corresponding author: MJ Nanjan, University of Madras, Vijayanagar Palace Road, Ootacamund 643001, Nilgiris, Tamilnadu, India, Tel: 0423 2450095; +919443220739;

\section{Editorial}

Volume 2 Issue 2

Received Date: May 03, 2018

Published Date: May 10, 2018

Email: mjnanjan@gmail.com

\section{Editorial}

Herbal drugs are finished and labeled medicinal products that contain aerial or underground plant parts or combinations thereof, whether in the crude state or as plant preparations, as active ingredients. Plant material includes juices, gums, fatty acids, essential oils, or other substances of this nature. Herbal drugs may contain excipients in addition to active ingredients.

The problem with herbal drugs is that they often consist of mixtures of different substances and this makes it difficult to subject them to analysis by the methods of modern science or to meet the regulatory and approval mechanisms that are designed for single molecules of modern medicine. Further, the quality of herbal drugs seems to mean different things to different people. Some equate quality with efficacy and/or potency. Others focus on the relationship between quality and safety. Still others focus on the marker content. The consensus today on the quality of herbal drugs seems to have been focused on the issue of the marker content. Further, efficacy and potency of herbal drugs are frequently used interchangeably. Efficacy is a qualitative term defined as the power to produce an effect whereas potency is a quantitative term defined as the amount of the active ingredient/drug required to produce a therapeutic effect. Although potency is often considered to be a quantitative measure of efficacy, if chemical assays are used to measure potency, then a product may be extremely potent but fail to be efficacious, if a sub therapeutic dosage schedule is used, if it is not available and if it is neutralized by other ingredients in the body.

Marker compounds are one or more constituents that occur naturally in the medicinal plants that are selected for special attention by the researcher or the manufacturer. The presence of the predetermined amount of marker compound(s), however, does not guarantee the potency of a herbal drug. The term potency requires biological assessment of an extract and cannot be determined solely by marker compound(s) measurement.

The concept of standardization of plant products was introduced in 1994 when the US Congress proposed Dietary Supplement Health and Education Act (DSHEA). Standardization is expected to guarantee therapeutically effective doses of stable and uniform products that maintain consistency from batch to batch. One can achieve the best possible control in double blind clinical studies. Translating this concept to herbal drugs is not as simple as it may appear because the content of botanical preparations depends upon, growing conditions, drying, climate, powdering, soil quality, extraction, time of harvest, etc.

But then what is standardization? Herbalists/traditional practitioners do not believe in standardization. They say standardization is chemicalization and pharmaceuticalization. They ask should nature be standardized. Should we not allow nature to continue its role? Nutrition experts do not insist that every carrot should contain the same amount of $\beta$ Carotene before recommending the same as a source of vitamin A. Standardization seems to be a controversial subject because some people define standardization is manufacturing a product to contain a specific amount of the herb in a dosage unit and others talk about consistent manufacturing process. The consensus today on standardization is manufacturing a product to contain a certain marker compound to a certain specified level Why marker concept? There are several factors that affect the 


\section{Journal of Natural \& Ayurvedic Medicine}

ultimate chemical profile of a botanical like genetics, growing conditions, harvesting, drying, storage, etc. With the current technology it is not possible to quantify the hundreds of chemical constituents in a timely and cost effective manner. The compromise solution to this dilemma is to select marker(s) and ensure that each batch contains the same amount of marker(s). This approach makes the assumption that the contents of other constituents will vary in proportion to the marker(s). The selection of marker compound(s) is based on the stability of the constituent, technical ease of analysis, utility to identify the plant, indicator of the product quality and the potential relevance to therapeutic effects.

As per WHO/EC guidelines there are different type of markers depending upon its desired role; active principle(s) are compounds with known pharmacological activity, chemically well- defined and accepted as the contributor for the therapeutic effect, active marker(s) are pharmacologically relevant, chemically well-defined and contribute to efficacy but lacking in proof that they alone are responsible for clinical efficacy, analytical marker(s) are characteristic compounds/major constituents for which content ranges are specified and negative marker(s) are used to screen for the presence of toxic/undesirable botanicals as well as unwanted constituents.

But the quality of a herbal drug based on the marker concept is questionable. Manufacturers normally ensure that a minimum amount of a marker, specified on the label is, present in a product. The product thus may contain marker(s) greater than the label claim. This defeats the very purpose of standardization to produce products that are consistent in strength. Further, the over emphasis on marker content has been a boon for unethical business men. It is easier to pass off adulterated products that only assess marker content. As per WHO however, standardization refers to the whole body of information and controls required to produce a formulation of reasonable consistency. This is achieved through minimizing the inherent variation in the composition of the natural product through quality assurance practices applied to medicinal plant growing, extraction and formulation development.

Scientific validation should be the key to standardization. The product should be therapeutically effective as claimed, safe (toxicological studies) and free from harmful contaminants like heavy metals, microbial, pesticides etc. The key to achieve the above is to carry out well designed, randomized, double-blind, placebo-controlled clinical studies on humans, determine the activity by pharmacological and clinical methods and prepare a qualitative and quantitative chemical profile of all the significant chemical constituents to establish phytoequivalence, establish the bioavailability of at least the major constituents and establish the dosage form composition by carefully selecting the excipients and other diluents. 